\title{
A Novel Approach for Automatic Timetable Generation
}

\author{
Mayuri R. Bagul \\ Student, Computer Engg, \\ BVCOE \& RI, Nashik \\ Savitribai Phule Pune \\ University
}

\author{
Sunil C. Chaudhari \\ Student, Computer Engg, \\ BVCOE \& RI, Nashik \\ Savitribai Phule Pune \\ University
}

\author{
Sunita N. Nagare \\ Student, Computer Engg, \\ BVCOE \& RI, Nashik \\ Savitribai Phule Pune \\ University
}

\author{
Pushkar R. Patil \\ Student, Computer Engg, \\ BVCOE \& RI, Nashik \\ Savitribai Phule Pune University
}

\author{
K.S. Kumavat \\ H. O. D., IT Dept., \\ BVCOE \& RI, Nashik \\ Savitribai Phule Pune University
}

\begin{abstract}
The manual system of preparing time table in colleges is very time consuming and tedious task which usually ends up with various classes clashing either at identical room or with same teachers having more than one class at a time. Due to manual approach, proper use of resources is neither effective nor efficient. To overcome all these problems we propose to make an automated system with computer assisted timetable generator. The system will take various inputs like number of subjects, teachers, maximal lectures a teacher can conduct, priority of subject and topics to be covered in a week or a lecture, depending upon these inputs it will generate possible time tables for working days of the week, making optimal use of all resources in a way that will best suit the constraints. An appropriate timetable is then chosen from the optimal solutions generated.
\end{abstract}

\section{Keywords}

Component, Constraints, Resource Scheduling Algorithm, Time Table generation.

\section{INTRODUCTION}

The class timetabling difficulty could be a planning formula with nice interest and implications within the fields of operational analysis and AI. Most institutes subsume this drawback manually, i.e. an effort and error methodology is employed to line a timetable. whereas setting a timetable, importance is given to effective utilization of re that becomes a really exhausting task that has to be self-addressed a minimum of once a year by each educational institute.

\subsection{Required}

The schedule should meet the necessity of latest course addition and recently listed students to recent batches. this might end in schedule the whole plan yet again for its entire batches and to be regular in shortest doable time before the batches course begin. Another drawback that happens whereas planning plan for exams, once multiple batches have examination on same day, they have to be schedules effectively taking under consideration all issues associated with facilities that area unit on the market to conduct these exams at the same time. The teaching staffs sometimes pay lots of your time in timetable generation and timetable management. the target of the Timetable Generator project was to develop a tool that permits institutes to dynamically generate timetables for colleges' internet access directly from "raw" schedule. This timetable generation code conjointly regard the supply of lecturers and different re whereas making timetable. Moreover, changes are often merely created within the timetable as and once necessary counting on the supply of lecturers, replacement, students, technicians, school rooms and lessons. Timetabling issues area unit a particular kind of planning drawback and area unit in the main involved with the assignment of events to slots subject to constraints with the resultant answer constituting a timetable. Wren (1996) demarcated timetabling as: "Timetabling is that the allocation, subject to constraints, of given to things being placed in house time, in such the way on appease as nearly as doable a collection of covetable objectives." The constraints in timetabling are often divided into 2 categories: exhausting and soft. exhausting constraints can not be profaned. Soft constraints don't seem to be imperative however their satisfaction is additional covetable so as to supply a decent quality timetable. A general timetabling drawback made from distribution variety of events like course, examinations, lectures, work meeting etc. into a restricted range of slots and rooms whereas minimising the violations of a collection of constraints. Heuristic optimisation ways area unit expressly aimed toward good possible answers which will not be optimum wherever complication of restricted time on the market doesn't enable actual solution. The empirical analysis of heuristic methodology is predicated on analytic drawback concerned within the tough worst case result. In its simplest kind the planning task made from mapping category, teacher and area combos onto time slots.

Time Table creation is exhausting and time consuming process for the faculty in charge. At present this is done manually as there are no efficient time table generators. While framing time table the basic problems are slot clashes. Allotting periods itself is so tedious that allotting the whole time table is not at all efficient when done manually. So, even the software which has already been created does not comply with the constraints. The present system is therefore time consuming, tedious process requiring manual labor and simultaneously, having less flexibility. Therefore we are proposing a system for Automatic timetable generation. The algorithm based application allots periods in such a way that no period clashing or faculty period clash is met. The subjects are paired in such a way that teachers remain associated and no period clash appears. Subjects are allotted as per priority based on the number of lectures per week of that subject. High priority subjects are given preference for number of periods 
per week and are allotted accordingly. Also the corresponding labs are allotted for the suitable day. This helps satisfy the constraint of number of subjects per day both theory and lab for a faculty. We assign subjects to teacher as per their seniority and as per their preference. This is done starting from the high priority subject first and then the decreasing priority subjects and then the unassigned subjects. This keeps utmost care of the designation of faculties and their $\mathrm{p}$. This procedure creates not only a feasible Time-Table for the department but also an optimal one. Output of the application is not only the class time table but also faculty timetable, both the optimum ones. The proposed system is based on heuristic algorithm that takes values and manages the constraints and source scheduling one by one. The system generates a timetable for the lecture courses as well as the staff timetable. It distributes workload of lectures equally among all the specified time slots. It prioritizes the lectures according to customized antecedence. If lecture cannot be adjusted then it can be moved up in higher priority slot until adjusted accordingly. In our system we have also implemented features other than the Timetable generation, such as a forum for student-teacher interaction, where students can put their queries and teachers can respond. Also teachers can upload ebooks and presentations for the students. This will prove quite helpful for the students as they can have access to these study materials any time, any place and get their queries solved and refer to those anytime again. Also students will be able to provide reviews for all the lectures and teachers. This will be helpful in getting an idea whether the timetable is being followed and how do the students rate the lectures based on their understanding of the subjects and topics taught during those lectures. These reviews will also be helpful for the staff to understand to what extend do the students comprehend and what topics they find difficult to understand during the lectures. Then the teachers will be able to accordingly schedule and reschedule the lectures for the students.

\section{LITERATURE SURVEY}

In some years two main approaches have been successful for implementing the timetabling problem. The first approach is based on local search procedures method such as simulated annealing, tabu search and genetic algorithms. These methods express constraints as some value of functions, which are minimized by a heuristic search of better solutions in reference of some initial feasible solution. The second approach is based on constraint programming (CP). Its main advantage is declaratively a direct statement of the constraints serves as part of the program. This makes the program easy to modify, which is critical in timetabling problems. The constraints are handled through a system of constraint propagation, which decrease domains of variables, coupled with backtracking search. The main disadvantages of these approaches are

1. Difficulties with expressing hard and soft constraints,

2. The need to determine their parameters through experimentation and

3. Possible problems with improving the initial feasible solution,

Which - as a rule - may be determined without problem? An attempt to overcome the drawbacks along soft constraints was discussed, successfully combined local search with constraint satisfaction to decrease their drawbacks. A custom-tailored distribution strategy is able to introduce soft constraints during a search, leading immediately to a "good" timetable; giving the ability to effectively optimize the timetable.
Bhaduri A [1], evolutionary techniques have been used to solve the time table scheduling problem. Methodologies like Genetic Algorithms, Evolutionary Algorithms etc have been used with mixed success. In this paper, we have reviewed the problem of educational time table scheduling with genetic algorithm. We have further solved the problem with a mimetic hybrid algorithm, genetic artificial immune network and compare the result with that obtained from genetic algorithm. Results show that GAIN is able to reach the ideal feasible solution faster than that of GA.

Dipti Shrinivasan [2], Find a feasible tutorial timetable in a large university department is a challenging problem faced repeatedly in educational establishments. This paper represents an evolutionary algorithm (EA) based approach to solving a strong constrained university timetabling problem. The move toward a problem-specific chromosome representation. Heuristics and also context-based reasoning have been used for received feasible timetables in a reasonable computing time. An intelligent flexible mutation scheme has been employed for fast-moving up the convergence. The comprehensive course timetabling system presented in this paper has been approved, tested and discussed using real world data from a large university.

Anuja Chowdhary [3] introduces a practical timetabling algorithm capable of taking care of both strong and weak constraints effectively, used in an automatic timetabling system. So that each teacher and student can view their timetable once they are final for a given semester but they do not edit them. Timetable Generation System generates timetable for every class and teacher, in keeping with the convenience calendar of teachers, availability and capacity of physical sources and some rules applicable at distinct classes, semesters, teachers and subject's level.

Anirudha Nanda [4], proposes a general solution for the timetabling problem. Most heuristic proposed previous approaches the difficulties from the students' point of view. This solution, however, works from the lecture's point of view i.e. lecturer availability for a given time slot. While all the strong constraints (e.g. the availability of teachers, etc.) are resolved rigorously, the scheduling solution presented in this paper is a flexible one, with a primary aim to solve the issues of clashes of lectures and subjects, pertaining to teachers.

A.Elkhyari [5], the proposed algorithm aids solving the timetabling problem while giving import to teacher availability. This algorithm uses a heuristic approach to give a overall solution to school timetabling difficulties. It initially uses randomly generated subject sequence to make a temporary time table. If the teacher has been apportion more than the allowed maximum lectures the subjects is moved toward into a Clash data structure. To avoid cycling to improve the search, this variable selection criterion can be randomized.

\section{[6], Harmony search algorithm (HSA) is a meta-heuristic}

Population-based method that is developed by Geem et al.

[7], the meta-heuristics were classified based on the number of solutions that are used at the same time into local searchbased (or trajectory) methods (i.e., hill climbing, tabu search, simulated annealing) and population-based methods (i.e., genetic algorithm, harmony search algorithm, ant colony optimization). 
[8] The MA has come to light as population-based metaheuristic algorithm by means of hybridizing the natural(population-based) and cultural (local search-based) selection Principles drawing from the principles of natural selection and the notion of an meme.

[9] In a recent comprehensive survey to examine timetabling, $\mathrm{Qu}$ et al. suggested "There are many research directions generated by considering the hybridization of meta-heuristic methods particularly between population-based methods and other approaches."

\section{SYSTEM OVERVIEW}

The block diagram of the proposed system shows the inputs provided to the system and the outputs which will be then used to assign constraint and get an optimal timetable as solution.

Figure 1 shows system overview which contains all details regarding system working. In system three users are there in which HOD can work as a admin which can monitor all communication between staff and student as well as all data on website.

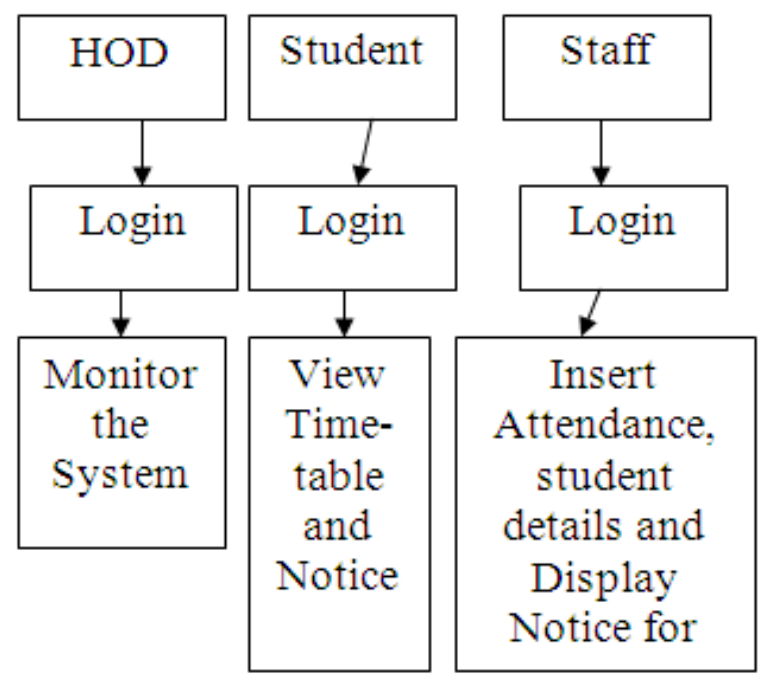

Fig 1: System Overview Diagram

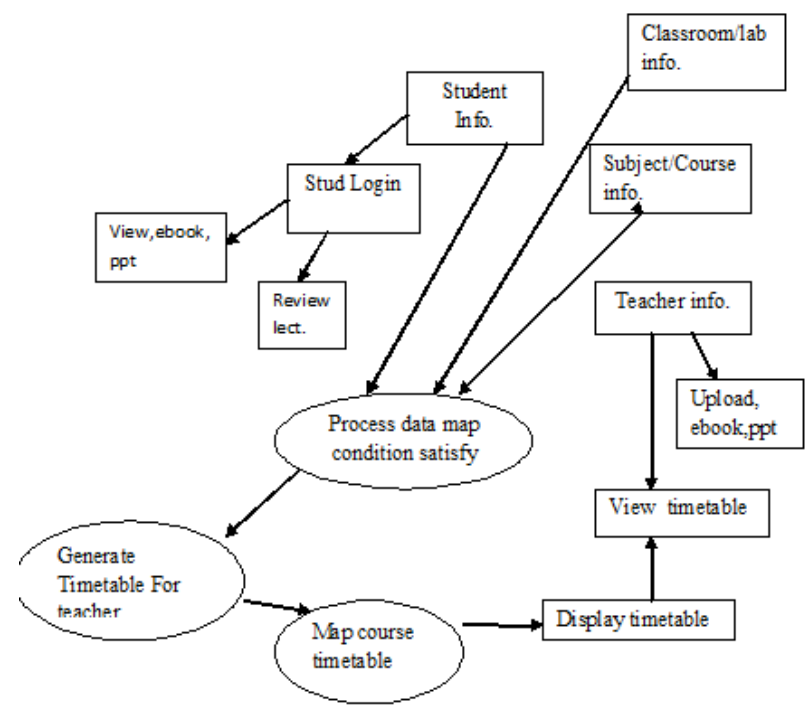

Fig 2: System Architecture
Figure 2 contain following things:

1. Enter Classroom Details, teacher details, Lab Details as classroom information.

2. Student details as student information, Subject details as subject information.

3. Teacher details as teacher information.

4. Process Data map and Conditions to satisfy: Process all the information as per HEURISTIC Algorithm.try to satisfy constraints accordingly depending upon priorities associated. Course Information can be viewed at this stage.

5. Generate timetable for teachers: Generated timetable satisfying hard constraints is to be viewed by teachers and define timetable that will try to satisfy maximum soft constraints.

6. Map course Timetable: After defining a timetable it will try to map timetable for whole course.

7. View Timetable: Timetable will be available for display, update for staff and teachers.

\section{MATHEMATICAL MODEL}

\subsection{Parameters}

There are individualizing constraints to be satisfied at the time to instantiate movables about time slots and classrooms. The constraints can be hierarchical into strong and weak constraints as follows:

\subsubsection{Strong Constraints}

$\mathrm{C} 1$ : A classroom is not assigned to many lecture at the same time.

$\mathrm{C} 2$ : An teacher cannot teach more than one class at the same time.

C3: Courses for the same year-meeting students of a department cannot take place at a time.

C4: The classroom for a course should have sufficient capability to take students registered in the course.

C5: The classroom should be well equipped with optional facilities for the classes.

\subsubsection{Weak Constraints}

C6: The lectures are not assigned to slots which are in the instructor's taboo time zones.

C7: teachers daily lecture hours should be limited to be within the allowed maximum hours.

C8: classes are scheduled in the teachers preferred time zones.

C9: A lunch break must be scheduled.

C10: If possible, the lectures should not be scheduled on night time slots.

C11: The theory subjects are scheduled on Monday and Tuesday, and the practical courses are scheduled on Wednesday, Thursday, and Friday.

C12: For daytime students, the cultural subjects are scheduled in the morning time slots (1st to 4th time slots on weekdays), and major subjects are scheduled in the afternoon time slots (5th to 8th time slots). 
C13: For night time students, the cultural courses are scheduled on the 11th to 12 th slots, and the major subjectscourses are scheduled on the 13th to 16th slots on weekdays.

C14: If possible, the lecture hours for a course should be scheduled continuously.

C15: As far as possible, classes should be scheduled in their corresponding department's reliable sources-use classrooms.

C16: The classrooms should be Assigning in a same manner to minimize the distances between adjacent classes' classrooms.

It is coveted for timetables to satisfy all strong and weak constraints. However, it is usually not simply to meet all these constraints. The virile constraints must be satisfied all the times, but impotent constraints can be somewhat sacrificed to find practice timetables. Among the weak constraints, constraints from $\mathrm{C} 6$ to $\mathrm{C} 14$ are imposed on the allocation of slots. Constraints $\mathrm{C} 15$ and $\mathrm{C} 16$ are imposed on the allocation of classrooms. The constraints are arranged in the sequence of importance in the scheduling. For example, if it is unthinkable to find schedules satisfying both C6 and C7 simultaneously, it is preferred to select a schedule that satisfies $\mathrm{C} 6$ but $\mathrm{C} 7$ rather than a schedule satisfying C7 but C6.

\section{ALGORITHMIC STRATEGY}

Algorithmic strategy contains heuristic algorithm, variable used for that and different assumption for satisfying goals:

\subsection{Heuristic Algorithm}

The term heuristic is used for algorithms which find out solutions among all possible ones, but they do not confirm that the best will be found, they may be thought as about and not accurate algorithms. Thesealgorithms, usually find a solution close to the best and they find it fast and simply. Sometimes these algorithms can be accurate, that is they defector find the best solution, but the algorithm is motionless called heuristic until this best solution is proven to be the best. The method old from a heuristic algorithm is one of the known methods, such as greediness, but in order to be simple and fast the algorithm ignores or uneven suppresses some of the problem's demands.

Consider the example of automatic timetable generator.

\subsection{VARIABLE USED:-}

--Time slots of the time tables:- ts1, ts2, ts $3 \ldots . .$, tsn

--List of Subjects:- s1,s2,s3, ..., sn

--Teachers: - $\mathrm{t} 1, \mathrm{t} 2, \mathrm{t} 3, \ldots . ., \mathrm{tn}$

--Batches of students:- c1,c2,c3, ....., cn

--Flags indicating finalized timeslots :- tsf1,

tsf 2, tsf $3, \ldots . .$, tsfn

--Data structure to hold Final Timetable:-final_tt

--Count for day of week: Daycount

--Number of days of the week:- $n$

--Data structure to hold Subject-clash within the day:- clash

--Each element of Clash data structure:-clash_element

--Data structure for Subject-clash across days:-Dayclash

--Each element of Dayclash data structure:-day_clash_element

--Subject contained in dayclash:-sdc
--Teacher associated with subject in dayclash:-tdc

--Max number of lectures of subject si in the week:-k

--Counter for the number of subjects:-counter_sub

--Random number indicating random slot allotment for subject:-rand_sub_allot

--Data structure to hold randomly allotted subject:rand_sub_seq

--Data structure to hold all subjects:-init_sub

\subsection{ASSUMPTION:-}

This algorithm is designed to solve and generate school time tables. The following is a list of assumptions made while developing this algorithm:

- The algorithm produces Ideal outputs in a five-day week.

- The number of subjects $(\mathrm{s} 1, \mathrm{~s} 2, \ldots, \mathrm{sn})$ need to be finalized before the algorithm begins execution.

- Number of teachers $(\mathrm{t} 1, \mathrm{t} 2, \ldots, \mathrm{tn})$ entered before execution of the algorithm are assumed to be constant and cannot be changed during or after the algorithm has been executed.

- Any change in the above two supposition will require a new generation of Timetable for the changed data.

- In each time table, all time-slot is occupied with, a unique combination of subjects without any repetition of subjects.

- Any teacher is allowed at most ' $k$ ' number of lectures in a week. The value of $\mathrm{k}$ is accepted before execution of the algorithm.

- It is assumed that a lecture cannot take more than one lecture for the same class in a day.

- Timeslots ts1, ts2, ..,tsn once entered at the beginning cannot be changed throughout the execution.

\subsection{Some Important Expected Results:}

Expected result for the system is shown in figure 3 as follows-

\begin{tabular}{|l|l|l|l|l|l|}
\hline & Mon & Tue & Wed & Thurs & Fri \\
\hline TS1 & T1,S1 & T1,S2 & T2,S3 & T3,S4 & T4,S5 \\
\hline TS2 & T4,S5 & T1,S1 & T1,S2 & T2,S3 & T3,S4 \\
\hline TS3 & T3,S4 & T4,S5 & T1,S1 & T1,S2 & T2,S3 \\
\hline TS4 & T2,S3 & T3,S4 & T4,S5 & T1,S1 & T1,S2 \\
\hline TS5 & T1,S2 & T2,S3 & T3,S4 & T4,S5 & T1,S1 \\
& & & & & \\
\hline
\end{tabular}

Fig 3: Expected Result Format

\section{CONCLUSION}

This application can alter the method of your time table generation swimmingly which can otherwise required to done victimization unfold sheet manually presumably resulting in constraints hassle that square measure tough to see once list is 
generated manually. The purpose of the rule to get a timetable schedule mechanically is happy. The rule incorporates variety of methods, aimed to enhance the potency of the search operation. It also, addresses the necessary exhausting constraint of clashes during the supply of lecturers. The nonstiff soft constraints i.e. optimization subjective for the search operation also are effectively handled. Taken the generality of the rule operation, it will additional be tailored to a lot of specific eventualities, e.g. School, examination planning and additional be increased to make railway time tables. Thus, through the method of automation of the time-table drawback, several an-hours of making a good timetable are decreased eventually. The foremost fascinating future directions within the development of the rule arise in its extension to constraint propagation. Once there's a price appointed to a variable, such assignment is propagated to unassigned variables to allow all values that get struggle with the present assignments. the data concerning such prohibited worth's is propagated furthermore.

\section{REFERENCES}

[1] Bhaduri a "university timetable scheduling using genetic algorithm".Advancesin Recent Technologies in Communication and Computing, 2009. ARTCom '09. International Conference

[2] DiptiShrinivasan "automated time table generation using multiple context reasoning for university modules" Published in: evolutionary computation, 2002. cec '02. Proceedings of the 2002 congress on (volume:2 )

[3] AnujaChowdhary "TIME TABLE GENERATION SYSTEM”.Vol.3 Issue.2, February- 2014, pg. 410-414

[4] Anirudha Nanda "An Algorithm to Automatically Generate Schedule for School Lectures Using a Heuristic Approach". International Journal of Machine Learning and Computing, Vol. 2, No. 4, August 2012.

[5] A. Elkhyari, C. Gu'eret, and N. Jussien, "Solving dynamic timetabling problems as dynamic resource constrained project scheduling problems using new constraint programming tools. In Edmund Burke and Patrick De Causmaecker, editors, Practice And Theory of Automated Timetabling, Selected Revised Papers," pp. 39-59. Springer- Verlag LNCS 2740, 2003.

[6] Z.W. Geem, J. H. Kim, and G. V. Loganathan, "A new heuristic optimization algorithm: Harmony search," Simulation, vol. 76, no. 2, pp. 60-68,2001.
[7] C. Blum and A. Roli, "Metaheuristics in combinatorial optimization: Overview and conceptual comparison," ACM Comput. Surv., vol. 35, no. 3, pp. 268-308, 2003.

[8] Y.-S. Ong, M.-H. Lim, N. Zhu, and K.-W. Wong, "Classification of adaptive memetic algorithms: A comparative study," IEEE Trans. Syst., Man, Cybern. B, Cybern., vol. 36, no. 1, pp. 141-152, Feb. 2006.

[9] R. Qu, E. K. Burke, B. McCollum, L. T. G. Merlot, and S. Y. Lee, "A survey of search methodologies and automated system development for examination timetabling," J. Schedul., vol. 12, no. 1, pp. 55-89, 2009.

\section{AUTHOR'S PROFILE}

Mayuri R. Bagul she is Engineering student of computer Engineering at Brahma Valley College of Engineering And Research Institute, Nasik under Savitribai Phule Pune University. Her interest in the field of Data analysis and programmer.

Sunil C. Chaudhari he is student of Engineering student of computer Engineering at Brahma Valley College of Engineering And Research Institute, Nasik under Savitribai Phule Pune University. His interest in the field of Application intelligence.

Sunita N. Nagare she is student of Engineering student of computer Engineering at Brahma Valley College of Engineering And Research Institute, Nasik under Savitribai Phule Pune University. Her interest in the field of Application intelligence.

Pushkar R. Patil he is student of Engineering student of computer Engineering at Brahma Valley College of Engineering And Research Institute, Nasik under Savitribai Phule Pune University. His interest in the field of Data analysis and programmer.

K. S. Kumavat, ME, BE Computer Engg. Was educated at Pune University. Presently she is working as Head Information Technology Department of Brahma Valley College of Engineering and Research Institute, Nasik, Maharashtra, India. She has presented papers at National and International conferences and also published papers in National and International Journals on various aspects of Computer Engineering and Networks. Her areas of interest include Computer Networks Security and Advance Database. 